EPJ B 15, $701(2000)$

\title{
Conserved Dynamics and Interface Roughening in Spontaneous Imbibition : A Phase Field Model
}

\author{
M. Dubé ${ }^{1,2}$ a, M. Rost ${ }^{1,2}$, K. R. Elder ${ }^{3}$, M. Alava ${ }^{2}$, S. Majaniemi ${ }^{1,2}$ and T. Ala-Nissila ${ }^{1,2,4}$ \\ 1 Helsinki Institute of Physics, P.O. Box 9, FIN-00014, University of Helsinki, Helsinki, Finland \\ ${ }^{2}$ Laboratory of Physics, P.O. Box 1100, Helsinki University of Technology, FIN-02015 HUT, Espoo, Finland \\ 3 Department of Physics, Oakland University, Rochester, MI 48309-4487, U.S.A. \\ 4 Department of Physics, Brown University, Providence, RI 02912-1843, U.S.A.
}

\begin{abstract}
The propagation and roughening of a fluid-gas interface through a disordered medium in the case of capillary driven spontaneous imbibition is considered. The system is described by a conserved (model B) phase-field model, with the structure of the disordered medium appearing as a quenched random field $\alpha(\mathbf{x})$. The flow of liquid into the medium is obtained by imposing a non-equilibrium boundary condition on the chemical potential, which reproduces Washburn's equation $H \sim t^{1 / 2}$ for the slowing down motion of the average interface position $H$. The interface is found to be superrough, with global roughness exponent $\chi \approx 1.25$, indicating anomalous scaling. The spatial extent of the roughness is determined by a length scale $\xi_{\times} \sim H^{1 / 2}$ arising from the conservation law. The interface advances by avalanche motion, which causes temporal multiscaling and qualitatively reproduces the experimental results of Horváth and Stanley [Phys. Rev. E 525166 (1995)] on the temporal scaling of the interface.
\end{abstract}

PACS. 47.55.Mh Flows through porous media - 05.40+j Fluctuation phenomena, random processes, and Brownian motion - 68.35.Ct Interface structure and roughness

\section{Introduction}

The dynamics of driven interfaces in disordered media is a subject of intense interest in nonequilibrium statistical mechanics. It is well established that for sufficiently strong driving, the interface feels an effective smeared out "thermal" noise and its fluctuations present all the typical phenomena of scale invariance of driven systems 11. In the opposite case of weak driving, the quenched nature of the noise becomes apparent and the interface may reach a ' pinned state, characterised by completely different scaling exponents [2].

An apparently easy experiment to perform is to monitor the motion of an invading liquid front in a porous medium. Many experiments have been done with Hele-

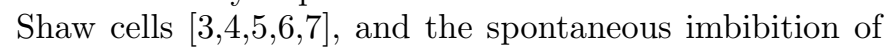
water in paper has also been considered [8, $9,10,11,12,13,14,15$ In this last case, capillary forces arising from the porous structure drive the liquid until loss of water by evaporation or hydrostatic pressure balance the driving. The eventual pinning of the front has received a lot of attention [8,9.12,14], and some experiments also examined the complete dynamical process [10,13, 15, 17]. It is generally believed that phenomena in the critical region of

a Present address: Center for the Physics of Materials, McGill University, 3600 rue University, Montréal, Québec, Canada H3A 2T8 the depinning transition can be described by local theories, i.e., the physics is governed by an equation that couples the interface locally with itself and the quenched randomness [2]. The spatial configurations of a pinned imbibition front seem to exhibit scaling properties well described by the "Directed Percolation Depinning" (DPD, or Quenched Kardar-Parisi-Zhang, QKPZ) universality class [8, 9, 12, 18]. An intuitively motivated lattice model of DPD compares well to experimental findings on the stopped front [8,9], and a modified version of the model addresses the influence of evaporation on the scaling properties of the pinned front [12].

However, these models neglect the fact that liquid has to be transported through the medium in order to drive the front, a nontrivial phenomenon in itself [19,20,21]. For example, viscous fluid transport explains why the invading

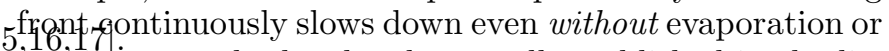
gravity, a result that has been well established in the literature 22,23.24]. In local models this has to be put in rather artificially 12 . The temporal correlations of the fluctuations should also reflect this nonlocality. It is thus doubtful whether any local model can explain the experimental results of Horváth and Stanley, focusing on the dynamical scaling of the interface 13].

The main concern in this paper is to analyse in detail a simple model of a propagating liquid-gas interface in a disordered medium, already introduced in Ref. [25]. The previous companion paper [24] presents a general 
Dubé et al.: Conserved Dynamics in Imbibition

overview of the experimental and macroscopic aspects of imbibition and discusses in particular the role of the fluid conservation law. The main goal in building the present model is thus to incorporate local liquid conservation to the interfacial dynamics. In Section 2 , it is shown that this can be achieved through a generalised Cahn-Hilliard equation. The model can then be applied to two different physical situations: a freely rising front, and a front propagating against a steady motion of the paper towards the liquid reservoir, leading to stationary fluctuations. A central feature of the model is the existence of a length scale $\xi_{\times}$, emerging from the interplay between interfacial tension and liquid conservation. This reflects the inherently non-local nature of the dynamics. The dynamical evolution of the model is numerically integrated in Section 3, for the two different setups. In Section 1 further implications of our modeling are discussed, and dynamical scaling in the experiments of Horváth and Stanley $\sqrt{13}$ is reinterpreted. We conclude with suggestions for future experimental work. The Appendices contain generalisations of the model to cases where gravity and/or evaporation might be important and a brief description of the steps required to obtain the interface equation, Eq. (9).

\section{Phase Field Model of Imbibition}

\subsection{Definition of the model}

A full model of the dynamics of a liquid invading a random medium based on a microscopic treatment is a formidable problem. The dynamics of the advancing interface between the liquid and the (dry) solid should however be amenable to a discussion at the coarse grained level. In this spirit, a phase-field model is used to represent the spatial configuration of wet and dry "phases". The field of interest is a locally conserved quantity $\phi(\mathbf{x}, t)$, defined on the half-plane $\{\mathbf{x} \equiv(x, y) \mid y \geq 0\}$, with values $\phi=+1(-1)$ for the wet (dry) phase. A free energy of the form $\mathcal{F}\{\phi\}=$ $\int d^{d} x\left[(\nabla \phi)^{2} / 2+V(\phi)\right]$ is chosen, with a double well potential $V(\phi)$ of the standard Ginzburg-Landau form, to which is added a linear tilt,

$$
V(\mathbf{x}, \phi(\mathbf{x}, t)) \equiv-\frac{1}{2} \phi^{2}(\mathbf{x}, t)+\frac{1}{4} \phi^{4}(\mathbf{x}, t)-\alpha(\mathbf{x}) \phi(\mathbf{x}, t) .
$$

The double well potential, together with the gradient term, ensures the existence of a well defined interface, and the quenched random field $\alpha(\mathbf{x})$ represents the random (coarse grained) structure of the medium. The first and the second moments associated with the underlying distribution of the random medium are given by $\langle\alpha(\mathbf{x})\rangle=\bar{\alpha}$, and $\left\langle\alpha(\mathbf{x}) \alpha\left(\mathbf{x}^{\prime}\right)\right\rangle-\bar{\alpha}^{2}=(\Delta \alpha)^{2} \delta\left(\mathbf{x}-\mathbf{x}^{\prime}\right)$. It is thus assumed that $\alpha$ is spatially uncorrelated, which may be a good approximation in the case of ordinary paper (the areal mass density has only short-range correlations [26]).

The dynamics of the conserved variable $\phi(\mathbf{x}, t)$ is determined by a continuity equation $\partial_{t} \phi+\nabla \cdot \mathbf{j}=0$, where the current $\mathbf{j}(\mathbf{x}, t)=-\nabla \mu(\mathbf{x}, t)$ is related to the gradient of the chemical potential $\mu(\mathbf{x}, t) \equiv-\delta \mathcal{F} / \delta \phi(\mathbf{x}, t)$. The resulting equation of motion,

$$
\begin{aligned}
\partial_{t} \phi(\mathbf{x}, t) & =\nabla^{2} \mu(\mathbf{x}, t) \\
& =\nabla^{2}\left[-\phi(\mathbf{x}, t)+\phi^{3}(\mathbf{x}, t)-\nabla^{2} \phi(\mathbf{x}, t)-\alpha(\mathbf{x})\right],
\end{aligned}
$$

is essentially the Cahn-Hilliard equation [27], also used to study critical fluctuations and phase ordering in presence of a conservation law (model B dynamics [28,29]). The variable $\alpha(\mathbf{x})$ here plays the role of the local chemical potential at the interface thus controlling the flux.

In model B dynamics [29], the domain walls are driven by the difference between incoming and outgoing current $\mathbf{j}=-\nabla \mu$. In the sharp interface limit, and for a slowly moving front, $\mu(\mathbf{x}, t)$ changes quasistatically, always satisfying $\nabla^{2} \mu=0$ in the bulk, plus the appropriate boundary conditions. At the interface, $\mu$ must obey the Gibbs-Thomson boundary condition

$$
\left.\Delta \phi \mu\right|_{\text {int }}=\Delta V-\sigma \mathcal{K},
$$

where $\mathcal{K}$ is the curvature, $\sigma=2 \sqrt{2} / 3$ is the surface tension of the model, the miscibility gap $\Delta \phi=\phi_{+}-\phi_{-}$ and $\Delta V=V\left(\phi_{+}\right)-V\left(\phi_{-}\right)$. The quantities $\phi_{ \pm}$are the equilibrium values of the phase field, defined by the usual tangent construction 29,30]. The interface motion is then determined by the normal velocity $v_{n}=-\left.\partial_{n} \mu\right|_{-} ^{+}$.

\subsection{Freely rising and stationary fronts}

With appropriate boundary conditions, the model presented above can encompass the typical experimental setups of imbibition, as depicted in Fig. 1. These are such that the value of the chemical potential $\mu(x, y=0)=$ $\alpha_{0} \neq \bar{\alpha}$ is imposed at the bottom end while the top end of the system is kept dry (i.e., $\partial_{y} \mu(y \rightarrow \infty)=0$ and $\phi(y \rightarrow \infty)=-1)$. This concept can easily be explained by a simplified situation where the quenched field $\alpha(\mathbf{x})$ is homogeneous and equal to a constant $\bar{\alpha}$. In this case, an equilibrium interface would be obtained by letting $\mu=-\bar{\alpha}$ throughout the whole system, with $\phi_{ \pm}= \pm 1$. On the other hand, imposing the boundary condition $\mu(x, y=0)=\alpha_{0}$ creates an imbalance in the chemical potential causing the interface to advance. If the interface at time $t$ is at a height $H(t)$, the chemical potential, as given by the Laplace equation, is

$$
\begin{gathered}
\mu(\mathbf{x}, t)=\mu(y, t)=\alpha_{0}-\left(\bar{\alpha}-\alpha_{0}\right) \frac{y}{H(t)}, \text { for } y \leq H ; \\
\mu(y, t)=-\bar{\alpha}, \text { for } y>H,
\end{gathered}
$$

resulting in a time evolution

$$
\frac{d H(t)}{d t}=\frac{\bar{\alpha}-\alpha_{0}}{2 H(t)}
$$

Thus, the further the interface is from the reservoir, the smaller its velocity. This classical result, known as the

\footnotetext{
${ }^{1}$ This is valid only if $\bar{\alpha}-\alpha_{0} \ll 1$. A more complete expression is given in Appendix A.
} 
Washburn equation, is well established experimentally, although discrepancies may arise 22, 23, 24, 31.

A slight modification to the model can be used to reproduce the experimental setup of Horváth and Stanley [13. In their experiment, the interface is forced to stay at a fixed mean height $H$ by constantly pulling down the paper towards the reservoir of liquid. Within the phase field model it is easy to add a constant downward drift $\mathbf{v}=-v \hat{\mathbf{y}}$, so that

$$
\begin{array}{r}
\partial_{t} \phi(\mathbf{x}, t)+\mathbf{v} \cdot \nabla \phi(\mathbf{x}, t)= \\
\nabla^{2}\left[-\phi(\mathbf{x}, t)+\phi^{3}(\mathbf{x}, t)-\nabla^{2} \phi(\mathbf{x}, t)-\alpha(\mathbf{x}-\mathbf{v} t)\right]
\end{array}
$$

keeping the same boundary conditions as for the freely rising column. Thus the interface between the wet and the dry region is kept at a height $H$ where a rising interface would have a velocity $-\mathbf{v}$, or

$$
H=\frac{\bar{\alpha}-\alpha_{0}}{2 v} .
$$

\subsection{Equation of motion and correlation length}

In presence of quenched disorder, via the field $\alpha(\mathbf{x})$, the interface will start to roughen, as shown in Fig. 2. An immediately noticeable feature is that the interface looks extremely rough locally but appears smooth on large length scales. This should indeed be expected intuitively, since the physics of the phenomenon is such that parts of the interface ahead of the average interface position have a smaller instantaneous local velocity. They are thus eventually "caught up" by the average interface. Likewise, retarded parts of the interface tend to catch up with the average interface position. This idea is indeed confirmed by the numerical results, and can further be used to define the spatial range over which the correlated roughness may be seen.

A key step in understanding the physics consists in writing an interface equation for the present model. A single-valued one dimensional interface $y=h(x, t)$ is assumed, and the Green's function of the problem is defined through the relation

$$
\nabla^{2} G\left(x, y \mid x^{\prime}, y^{\prime}\right)=-\delta\left(x-x^{\prime}\right) \delta\left(y-y^{\prime}\right)
$$

for the range $-\infty<x, x^{\prime}<\infty, 0<y, y^{\prime}<\infty$, with Dirichlet boundary conditions. The half-plane must be used, since the presence of an "infinite reservoir" at position $y=0$ breaks the translational symmetry in $y$. Without any loss of generality, $\alpha_{0}=0$ is set from now on. The standard procedure 29,32,33, exposed in Appendix B, may then be followed to obtain the integro-differential equation of motion

$$
\begin{array}{r}
\int_{-\infty}^{\infty} d x^{\prime} G\left(x, h(x, t) \mid x^{\prime}, h\left(x^{\prime}, t\right)\right) \frac{\partial h\left(x^{\prime}, t\right)}{\partial t}= \\
\eta(x, h(x, t))+\sigma \mathcal{K}
\end{array}
$$

with the half-plane Green's function

$$
G\left(x, y \mid x^{\prime}, y^{\prime}\right)=\frac{1}{4 \pi} \ln \frac{\left(x-x^{\prime}\right)^{2}+\left(y-y^{\prime}\right)^{2}}{\left(x-x^{\prime}\right)^{2}+\left(y+y^{\prime}\right)^{2}}
$$

The quenched noise is written as $\eta(x, h) \equiv \int d y \phi_{0}^{\prime}(y-$ $h(x, t)) \alpha(x, y) \sim 2 \alpha(x, h)$ in the sharp interface limit. The Gibbs-Thomson boundary condition, $\left.\mu\right|_{\text {int }} \sim \mathcal{K}$ can be immediately obtained from Eq. (9) in the limit $\dot{h}=0$ since $\eta$ is the chemical potential at the interface. Analogous non-local equations will arise in the context of directional solidification, pattern selection in Laplacian fluid flow [34 and step growth [35]. The novel features here are the broken translational invariance and the presence of quenched noise. The interface fluctuations are thus intimately coupled to both the average position and the average velocity of the interface, a result that comes out self-consistently from the model. This is quite different from local types of equations or models. It should be particularly noted that the presence of a conservation law does not result in a "conserved" interfacial equation. Likewise, nonlinear equations with long range kernels [36] do not apply to the situation encountered here.

The difference between local models and Eq. (9) becomes even clearer if the interface is linearised in small deviations around the mean interface position $H(t)$ to obtain

$$
\begin{array}{r}
\dot{h_{k}}\left(1-e^{-2|k| H}\right)+|k| \dot{H} h_{k}\left(1+e^{-2|k| H}\right)= \\
|k|\left(\{\eta(t)\}_{k}-\sigma k^{2} h_{k}\right),
\end{array}
$$

where $h_{k}$ are the Fourier components of $h$ and $H=h_{0}$ is the average interface position. Note that the interface configuration enters the disorder term in Eq. (11) in a fundamentally nonlinear way,

$$
\{\eta(t)\}_{k} \equiv \int_{x} e^{-i k x} \eta(x, h(x, t))
$$

This equation already yields important information for the roughening process. For example, the limit $k \rightarrow 0$ reproduces the slowing down of the front as given by Eq. (5). It also reveals the different length scales in the problem. The average height of the interface separates two regimes of lateral scale. For $k H \ll 1$, Eq. (11) becomes

$$
\frac{d h_{k}}{d t}+\frac{1}{H} \frac{d H}{d t} h_{k}+\frac{\sigma}{2 H} k^{2} h_{k}=\frac{1}{2 H}\{\eta(t)\}_{k},
$$

and in the opposite short scale limit, $k H \gg 1$

$$
\frac{d h_{k}}{d t}+|k| \frac{d H}{d t} h_{k}+\sigma|k|^{3} h_{k}=k\{\eta(t)\}_{k} .
$$

The average interface height $H$ is thus also a lateral length scale. If two points are separated by a distance $r \gg$ $H$, they are not connected through the bulk of the system and receive liquid from the reservoir independently. In that sense, the dynamics of the interface on larger scales is local, although the slowing down of the interface is inherently a non-local phenomenon, reflecting liquid transport through the medium. In the opposite limit of $r \ll H$, the two interface points will be coupled through the bulk and compete for liquid coming from the same region behind the front. The dynamics of the interface then becomes fully non-local. 
However, in both limits the damping terms induce a separating length scale $\xi_{\times}=(\sigma / \dot{H})^{1 / 2}=(\sigma H / \bar{\alpha})^{1 / 2}$. For $\xi_{\times} k \gg 1$ the fluctuations of the interface are damped due to the line tension $\sigma$, while for $\xi_{\times} k \ll 1$, it is due to flow from the reservoir. By this mechanism, it is expected that the front is smoother on length scales larger than $\xi_{\times}$as compared to smaller scales.

This length scale is closely related to the Mullins-Sekerka instability of driven Laplacian fronts [37], although the situation is reversed here. Because fluid is transported towards the front from behind, advanced (retarded) parts of the interface receive less (more) mass than the average and the front is stabilised at long length scales. This result can intuitively be understood as follows. Due to the Gibbs-Thomson effect a local "bulge" of vertical extent $W$ and lateral size $\xi$ alters the chemical potential by $\Delta \mu \simeq \sigma W / \xi^{2}$. On the other hand, the average gradient in $\mu$ in the bulk liquid induces a difference $\Delta \mu \simeq \bar{\alpha} W / H$ across a vertical distance $W$. These two differences balance each other at a length given by

$$
\xi_{\times} \simeq \sqrt{\sigma H / \bar{\alpha}} .
$$

The length scale $\xi_{\times}$is a static quantity, but in a rising liquid column it becomes dynamical through the time dependence of $H(t)$, i.e., $\xi_{\times}=\xi_{\times}(t) \sim(H(t))^{1 / 2} \sim t^{1 / 4}$. It must also be noted that it is not a truly dynamical correlation length in the sense of kinetic roughening theories [1]. However, it is a time dependent upper cutoff for correlated fluctuations increasing with time, and therefore can be interpreted as a dynamic correlation length.

To draw any further analytical conclusions from Eq. (11) is extremely problematic due to the difficulties encountered with the quenched noise $\{\eta\}_{k}$ (which are analysed e.g. in [38]). Furthermore, Eq. (11) is a linear approximation to Eq. (9). Although the length scales come out correctly, it cannot be expected a priori that the correct scaling properties of the interface will be obtained. An analysis along the lines of Ref. [38] may prove insufficient, and simplified treatments, such as those attempted in Ref. 21] are inadequate.

\section{Numerical Analysis}

The interface fluctuations in the presence of quenched disorder were analysed by numerical integrations of Eq. (2) and Eq. (6). The position of the interface $h(x, t)$ at each $x$ was defined by the zero of the phase field, i.e. by $\phi(x, h(x, t))=0$ determined by linear interpolation between the points of the numerical grid. Overhangs, appearing for strong disorder but otherwise absent were ignored by taking the lowest or highest zero of $\phi$ above a given foot point $x$. No quantitative differences were seen between these two choices.

The disorder $\alpha(\mathbf{x})$ is an independently distributed random variable on each grid point, with mean $\bar{\alpha}$, standard deviation $\Delta \alpha$ and chosen from different types of distributions (gaussian, uniform on a finite interval, and exponential). Without loss of generality, the lower boundary condition is chosen such that $\mu(x, y=0)=0$, leading to $\phi(x, y=0)=\phi_{0}$, with $\phi_{0}$ the solution of $-\phi_{0}+\phi_{0}^{3}=\bar{\alpha}$.

To evaluate any scaling behaviour the first quantities of interest are the total width of the front,

$$
W^{2}(t)=\left\langle\overline{(h(x, t)-H(t))^{2}}\right\rangle,
$$

and the related spatial two-point correlation functions of the $q$ th moments

$$
G_{q}(r, t)=\left\langle\overline{|h(x+r, t)-h(x, t)|^{q}}\right\rangle^{1 / q} .
$$

The case $q=2$ is directly related to the structure factor $S(k, t)=\left\langle\overline{h_{k}(t) h_{-k}(t)}\right\rangle$. In the above equations the brackets denote an average over different realisations of $\alpha$, and the overbar a spatial average over the system. In presence of a stationary state also temporal averages can be taken.

The standard Family-Vicsek scaling assumption rests on a dynamical correlation length $\xi_{t} \sim t^{1 / z}$ where $z$ is the dynamical exponent, related to the decay of fluctuations along the interface. The maximal value it can attain is the system size $L$ at which point the interface is said to be in a "saturated" stationary state. The two-point correlation function then has a scaling form $G_{2}(r, t)=r^{\chi} f\left(r / \xi_{t}\right)$ where $f(u)=$ const. for $u \ll 1$ and $f(u) \sim u^{-\chi}$ for $u \gg 1$, a form which introduces the roughness exponent $\chi$ and defines the associated growth exponent $\beta=\chi / z$. The structure factor has a corresponding scaling form, $S(k, t)=s\left(k \xi_{t}\right) / k^{1+2 \chi}$ with the scaling function $s(u)$ constant for $u \gg 1$ and $s(u) \sim u^{1+2 \chi}$ for $u \ll 1$.

This picture may turn out to be incomplete or even wrong for the following reasons. First, the structure factor may contain an explicit time dependence besides $\xi_{t}$, $S(k, t) \sim t^{2 \kappa} s\left(k \xi_{t}\right) / k^{1+2 \chi}$, which is sometimes referred to as intrinsic anomalous scaling [39]. Second, if the interface is superrough, a case characterised by $\chi>1$, then $G_{2}(r, t) \sim \xi_{t}^{\chi}\left(r / \xi_{t}\right)^{\chi_{\text {loc }}}$ with a local exponent $\chi_{\text {loc }}=1$, since, by construction, $G_{2}(r)$ cannot increase faster than $r$ [40. In contrast to the standard Family-Vicsek picture, in both these cases the correlation function for $r<\xi_{t}$ do not saturate as long as $\xi_{t}$ increases [1]. This can be parametrised by the scaling of the local slopes $G_{2}(r=$ $1, t) \sim t^{\left(\chi-\chi_{\text {loc }}\right) / z}$. Third, the lateral $\xi_{t}$ and the vertical scale $W$ may not be enough to characterise the interface fluctuations, and different moments of $G_{q}$ may possess different scaling exponents $G_{q}(r) \sim r^{\chi_{q}}$ (see e.g. [41]).

The interface scaling behaviour may also be observed in the temporal correlation functions

$$
C_{q}(t)=\left\langle\overline{|h(x, t+s)-H(t+s)-h(x, s)+H(s)|^{q}}\right\rangle^{1 / q},
$$

which increase as $C_{q}(t) \sim t^{\beta_{q}}$ at short time differences $t$. Of course, this definition makes only sense in a steady state, under time-translational invariance. It therefore applies to the analysis of Eq. (6), where the average interface height is kept fixed by pulling down the paper at constant velocity $v$. 


\subsection{Freely rising fronts}

In this Subsection, the classic imbibition experiment is considered. A liquid front is allowed to invade the porous medium starting from a reservoir placed at $y=0$. Successive configurations obtained from numerical integration of Eq. (2) are presented in Fig. 2. The time difference between the curves is constant $\left(\Delta t=10^{3}\right)$, and the slowing down of the interface positions becomes apparent from the fact that they lie closer together the higher the front gets.

In the presence of quenched disorder $\alpha(\mathbf{x})$ the total width of the interface increases as a power of time. Fig. 3 shows that $W(t) \sim t^{\beta}$ with $\beta \approx 0.3$. In the same graph the progression of the average interface height $H$ is seen to follow Washburn's behaviour as expected from the analytic arguments.

It turns out to be impossible to determine the global roughness exponent $\chi$ from its definition in terms of the dependence of the saturated width on the lateral system size. Instead, the structure factor $S(k, t)$ is used. It is plotted on Fig. 4 for a system of size $L=256$, with $\bar{\alpha}=0.2$ and $\Delta \alpha=0.2$ at various times $10^{3}<t<5 \times 10^{4}$ (in the dimensionless units of Eq. (2)), corresponding to heights ranging from $20<H<100$. Although it is difficult to obtain good statistics for this quantity, immediately apparent is a strong power law decay, $S(k) \sim 1 / k^{1+2 \chi}$, with a global roughness exponent $\chi \approx 1.25$, and a crossover to a plateau corresponding to distances larger than the time-dependent correlation length $\xi_{\times}(t)$.

The form of the structure factor indicates the presence of anomalous scaling; with $\chi>1$ the surface is superrough [1] and the spatial correlation functions will reflect this fact. The level of $S(k, t)$ in the region of the power decay also seems to increase with time, i.e. $S(k, t) \sim t^{\theta} / k^{1+2 \chi}$, with $\theta \approx 0.05$, which could indicate the presence of intrinsic anomalous scaling [39]. A clear identification of this regime is however quite difficult, due to the very slow increase in time and to poor statistics.

The anomalous form of the scaling is most visible in the two-point spatial correlation function $G_{2}(r, t)$ as shown on Fig. 5 for the same data as for the structure factor. The correlated roughness of the interface is visible up to a length scale $r_{\max } \sim \xi_{\times}(t)$, and the average mean step height $G(r=1, t) \sim \xi_{\times}^{\chi-\chi_{\mathrm{loc}}} \sim t^{\left(\chi-\chi_{\mathrm{loc}}\right) / 4}[1,39]$.

The average driving force $\bar{\alpha}$ affects the scaling of the structure factor and correlation length only through the correlation length $\xi_{\times}(t) \sim(t / \bar{\alpha})^{1 / 4}$, and the total strength of the noise $\Delta \alpha$ only influences the amplitude of the prefactor of the correlation function. The correlation function may be fitted to the function

$$
G_{2}(r, t)=\Delta \alpha \xi_{\times}^{\chi} g\left(r / \xi_{\times}\right),
$$

with $\chi=1.25$ and a scaling function $g(x)=x^{\chi_{\text {loc }}} f(x)$ with $f(x) \sim x^{-\chi_{\text {loc }}}$ for $x \gg 1$, and approaching a constant for $x \ll 1$. There seems to be no simple explanation why $\Delta \alpha$ enters Eq. (19) in a linear way even beyond the linear approximation of Eqs. (13) and (14) where it is easy to see. The local scaling exponent $\chi_{\mathrm{loc}} \simeq 0.9$ is a direct con- sequence of anomalous scaling 2. Likewise, the structure factor may be described by the scaling form

$$
S(k, t)=\frac{s\left(k \xi_{\times}\right)}{k^{1+2 \chi}}
$$

where the scaling function $s(x)$ is constant for $x \gg 1$ and $s(x) \sim x^{1+2 \chi}$ for $x \ll 1$. The scaling behaviour can be seen in the inset of Fig. 5, where the scaled form of the correlation function is shown for a single system of $L=256$, with $\bar{\alpha}=\Delta \alpha=0.2$ at various times and in Fig. 6 where the scaled correlation function is now shown for systems of similar lateral extent but different values of the driving force and strength of disorder. Within this scaling picture, the early time development of the width follows $W(t) \sim \xi_{\times}^{\chi} \sim t^{\chi / 4} \equiv t^{\beta}$ yielding a growth exponent $\beta=$ $\chi / 4 \approx 0.31$ in good agreement with the direct numerical estimate.

\subsection{Fronts at Fixed Height}

The results presented in the last section indicate a scaling picture to be valid in the freely rising case. It is however difficult to obtain sufficient statistics and larger samples of quenched disorder replicae are necessary to get accurate data. This difficulty can be overcome by considering the stationary interface, as described by Eq. (6). In this case, the interface fluctuations reach a steady state and the various correlation functions can be obtained with greater accuracy. This setup also allows the investigation of height difference temporal correlation functions and permits a comparison with the experimental results of Horváth and Stanley 13].

Equation (6) was integrated numerically for different values of mean height $H=\bar{\alpha} / 2 v$ (see Eq. (7)). Different lateral system sizes were used between $L=12$ and $L=400$. The total vertical extent of the lattice was taken to be about 50 length units higher than the interface, to prevent any influence of the upper boundary. The mean value $\bar{\alpha}=0.2$ in all cases, but different distributions were used: (i) a uniform distribution with mean $\bar{\alpha}=0.2$, on the range $[0,0.4)$ (standard deviation $\Delta \alpha=0.07$ ), (ii) the same, but on the range $[0.1,0.3),(\Delta \alpha=0.03)$, (iii) an exponential distribution with average $0.2(\Delta \alpha=0.2)$. Different numbers of configurations were used in taking the averages, from 10 in the largest systems $(L=2 H=400)$ to 100 in the smallest $(L=2 H=50)$. Even in the largest systems, saturation of the interfacial fluctuations became apparent after times $t \simeq 2 \times 10^{4}$. All systems were integrated up to $t=10^{5}$, with the interface configurations extracted at time intervals $\Delta t=100$.

\subsubsection{Spatial correlations}

Along with the height $H$, the length scale $\xi_{\times}$also remains fixed in this setup, since it is related to the driving velocity through $\xi_{\times} \sim v^{-1 / 2} \sim H^{1 / 2}$. Thus, contrary to the

\footnotetext{
2 In principle $\chi_{\mathrm{loc}}=1$ for a superrough interface. However, here the scaling behavior in $S(k, t)$ appears only over a relatively short range and finite size effects are pronounced.
} 
standard picture of kinetic roughening, the saturation of the interface is not necessarily determined by the total lateral extent $L$ of the system. Here, the correlations saturate at either the system size $L$ or the correlation length $\xi_{\times}$, whichever is smaller. Fig. 77 shows data obtained for $H=50$ on system sizes $L=12,25,50,100,200$ and 400 . The curves for $L=100,200$ and 400 collapse indicating that the fluctuations are bounded by the $L$ independent length scale $\xi_{\times}\left(50<\xi_{\times}<100\right.$ for this particular case).

The structure factor $S(k, H)$, shown in Fig. 8, also has a pronounced power law decay $k^{-(2 \chi+1)}$ with a global roughness exponent $\chi=1.25$. Again, the interface is superrough. As in the freely rising case, there also seems to be a very weak intrinsic anomaly in the sense of [39], i.e. the prefactor of $S(k, H)$ in the power law region depends on $H$. The data are consistent with $H^{\theta}$ for $0 \leq \theta \leq 0.1$, but not accurate enough to draw any firm conclusion here.

Provided that $\xi_{\times}<L$ the spatial correlation function $G_{2}(r, H)$ follows the scaling form

$$
G_{2}(r, H)=\Delta \alpha v^{-\chi / 2} g\left(r v^{1 / 2}\right) .
$$

with the scaling function $g(x)$ defined as in Eq. (19). For all different setups, $L=50$ to $400, H=25$ to 200 , and all three choices for the disorder $\alpha(\mathbf{x}), G_{2}(r, H)$ is shown rescaled according to Eq. (21) in Fig. 10. For small distances $r<\xi_{\times}$the spatial correlation function is of the form $G_{2}(r) \sim r \xi_{\times}^{\chi-1}$, yielding a local roughness exponent $\chi_{\text {loc }} \approx 1$ and a height difference at fixed $r$ growing as $\xi_{\times}^{\chi-1}$ [1] (see Fig. 9).

Finally, the different moments $q=2,4$ and 6 of the correlation functions $G_{q}(r)$ can be compared, as shown in the inset of Fig. 9. All moments have a local exponent $\chi_{l o c} \sim 0.95$. The global exponent $\chi_{q}$ can in principle be obtained from the short distance scaling of $G_{q}(r=1) \sim$ $\xi_{\times}^{\chi_{q}-1}$. The present data point to a similar value $\chi_{q}=1.25$ for all $q^{\prime} s$ but are however too noisy to draw any definite conclusion. Thus, the interface is probably truly self-affine up to the crossover scale - be it the system size $L$ or the saturation length $\xi_{\times}$.

\subsubsection{Temporal correlations}

Before the interface fluctuations reach the steady state they are governed by an increasing dynamical correlation length. Starting from a flat front $h(x, t=0) \equiv H$ it is observed to grow roughly as $\xi_{t} \sim t^{1 / 3}$ and then approach $\xi_{\times}$. However, this behaviour could not be analysed in much detail, due to the short time range of the initial power law and to poorer statistics (averaging over time is not possible). The insets in Fig. 8 shows $S(k, t)$ approaching the saturated regime for the large system, $L=2 H=400$.

Next, the correlation functions $C_{2}(t)$, shown in Fig. 11 for different heights are compared, as in the experiments of Horváth and Stanley [13]. In both cases, the crossover time $t_{s}$ between the power law regime of $C_{2}(t)$ and saturation increases with $H$. This is true for the level of saturation (or the width $W$ ) as well. At early times $t \ll t_{s}$, the absolute value of $C_{2}(t)$ decreases with $H$.
The data can be related to a scaling function of the form

$$
C_{2}(t) \sim H^{\chi / 2} f\left(t / H^{z / 2}\right),
$$

with the scaling function $f(x) \sim x^{\beta_{2}}$ for $x \ll 1$ and const. for $x \gg 1$ with a (genuine) dynamical exponent $z \approx 2$ and the effective slope $\beta_{2} \approx 0.85$. Although the exact value of these exponents is difficult to establish, this form is however valid for all $L$ provided that $\xi_{\times}<L$.

The different moments $C_{q}(t)$ for $q=2,4$ and 6 are shown in Fig. 12. They clearly have different behaviour. The early time logarithmic slopes of the higher moments $\left(\beta_{q}\right)$ decrease with $q$, as shown in the inset of Fig. 12. For the higher moments, the effective exponents $\beta_{4} \approx 0.76$ and $\beta_{6} \approx 0.69$. Such multiscaling has been observed in cases connected with the existence of avalanches in the interface dynamics [42]. It is clear from Fig. 2 that similar avalanche type of motion exists here as well, but only up to the vertical length scale $W$ and lateral size $\xi_{x}$. Because of this reason the quantitative characterization of such avalanches is beyond the scope of the present work.

\section{Discussion}

\subsection{Temporal Scaling of the Interface and Relation to Experiments}

It is interesting to first compare the results for the stationary fronts with those of the freely rising fronts. Both cases are governed by the same height dependent length scale $\xi_{\times}$. In Fig. 13 the spatial correlation function $G_{2}(r, H)$ (fixed $H$ at saturation for the stationary case) and $G_{2}\left(r, t_{H}\right)$ (freely rising case at times $t_{H}=H^{2} / \bar{\alpha}$ when the average height has reached $H$ ) are shown for various values of $H$. There is a complete equivalence between the interfacial fluctuations at an instantaneous height $H(t)$ and the saturated fluctuations of a stationary interface. In both cases, the range of correlated roughness is determined by the same value $\xi_{\times}$. The length scale $\xi_{\times}$is thus conceptually different from the intrinsic time dependent lateral correlation length $\xi_{t}$ commonly found in models of kinetic roughening. Here $\xi_{\times}$merely fixes the maximum range of correlated roughness. Such "quasi-stationarity" of the moving front can only occur provided that the "natural" dynamical exponent $z<4$, so that the interface fluctuations can always catch up instantly with the available area of correlation.

The model can also help to interpret the experiments performed by Horváth and Stanley on the stationary interface [13], since it yields qualitatively similar results: The exponent $\beta_{2}$ is constant for all driving forces (and also $\beta_{q}$ for the higher moments considered), the level of saturation of $C_{2}(t)$ increases as $H$ increases, while the amplitude of the early time power law behaviour decreases.

Still, there is a quantitative difference between the experiments of 113] and the numerical results. In the present work, $\beta_{2}=0.85$ in contrast to the experimental value 0.56 . Clearly one would not expect coincidence, since already the average front velocity behaves differently: The model 
shows "pure" Washburn behaviour, $d H / d t \sim 1 / H$, whereas in $13 d H / d t \sim H^{-1.6}$. This deviation from Washburn's law occurs in many paper imbibition experiments [24], and there is no reason why its origin should not affect the intrinsic fluctuation dynamics as well, leading to different values of $\beta_{q}$ in the time correlation functions.

There is however a clear contradiction of the results with the scaling form for the time-correlation function suggested in [13],

$$
C_{2}(t) \sim V^{-\theta_{L}} L^{\chi} \mathcal{C}\left(t L^{-\chi / \beta} V^{\left(\theta_{t}+\theta_{L}\right) / \beta}\right)
$$

with the relation $V=d H / d t \sim H^{-1.6}$ and the scaling function $\mathcal{C}(u)$ such that $C_{2}(t) \sim t^{\beta} V^{\theta_{t}}$ if $u \ll 1$ and $C_{2}(t) \sim L^{\chi} V^{-\theta_{L}}$ in the opposite limit. Equation (23) does not include an explicit lateral length scale $\xi_{x}$, although some power of $V^{\theta_{t}+\theta_{L}}$ may obviously play such a role. This scaling form is however in contradiction with the present results in two ways. First, provided that $\xi_{\times}>L$, the total lateral system size does not play any role in the scaling of the interface. Secondly, no common scaling form interpolating from large systems (fluctuations up to scale $\xi_{\times}$) to small systems (dominated by $L$ ) has been found. The saturation of $C_{2}(t)$ is sharper for small $L$, which is visible in Fig. 11.

In the experiments only one lateral system size was used, so the role of the system size $L$ could not be assessed. Unfortunately, no information on the spatial scaling of fluctuations was presented so that no comparison can be done to the present work. Likewise, the higher moments of the temporal correlation function were not measured and the presence of avalanches could not be inferred.

\subsection{Avalanches, Pinning and Roughness of the Interface}

As one can see from Figure 2 the interface motion indicates the presence of "avalanches" as in usual models describing depinning transitions [2]. Here the behavior is however somewhat different since the flow of liquid in the average tends towards regions of lower chemical potential. In some way, this is analogous to 'self-organised' interface models in which the interface is driven at the point where the force is the largest $42,43,44$.

The question is now whether it is possible to understand the observed exponents - $\chi, \beta_{q}$ etc. - in terms of an avalanche description. Were this to be true, the presence of the conservation law would only be felt through the correlation length $\xi_{\times}$, which would limit the avalanche area to $\xi_{\times}^{\chi+1}$ with an exponential cut-off on the avalanche size distribution (see [7] for a discussion of a related experiment). It turns out that the multiscaling observed here $\left(\beta_{q}\right)$ differs in a crucial way from that obtained for avalanches in self-organised depinning. Leschhorn and Tang 42 obtain an almost trivial multifractal spectrum for the $\beta_{q}$ 's assuming that a local dynamical exponent $z_{\text {loc }}$ and a global roughness exponent can be defined. Dynamical scaling in this sense is however absent in the present case, since the interface dynamics depends directly on the local height.

The observed global roughness exponent $\chi=1.25$ also appears in "nonconserved" front propagation through a medium with quenched disorder 45, 46, 47, i.e. for interfaces in the quenched Edwards-Wilkinson (QEW) universality class. In that problem a similar value of $\chi$ is observed close to criticality, whereas a cross-over to thermal EW (the massless Gaussian field, with $\chi=0.5$ ) takes place in the moving phase. The imbibition model may then present a similar behaviour due to the continuous slowing down of the interface, with parts of the interface pinned (thus approaching the depinning transition from above). As in the $\mathrm{QEW}$ class, the value $\chi=1.25$ means that the width increases faster than the correlation length. The "local slopes", $G_{2}(r=1, t) \sim \xi_{\times}^{\chi-\chi_{\text {loc }}}(t)$ diverge with time and the local roughness exponent $\chi_{\text {loc }} \approx 0.9 \ldots 0.95$. For QEW models this is true on any scale - up to saturation in a finite system - but in our case the behavior can hold only up to $W \simeq \xi_{x}$, because the removal of overhangs occur naturally in a phase field formulation. If $W>\xi_{\times}$overhangs in neighboring bulges of the interface can merge, always keeping $\xi_{\times}$of the order of $W$.

A possible mechanism by which the roughness might change is if the interface becomes completely pinned. In Appendix A, it is shown that this may be achieved through the inclusion of either evaporation or gravity. In such a case, new length scales come into play, and the roughness exponent is most likely changed.

Although the pinning effects of evaporation have not been explicitly considered in this work, preliminary calculations show that evaporation, characterised by an evaporation rate $\epsilon$ introduced in the Appendix A, pins the interface at a height $H_{p} \sim(\bar{\alpha} / \epsilon)^{1 / 2}$. At this pinning height, a new correlation length $\xi_{\epsilon}$ emerges. For weak evaporation, defined as $\epsilon \ll \bar{\alpha}^{3} / \sigma^{2}$, the length $\xi_{\epsilon} \sim(\sigma / \epsilon)^{1 / 3} \gg$ $\xi_{\times}\left(H_{p}\right)$. For strong evaporation, $\epsilon \gg \bar{\alpha}^{3} / \sigma^{2}$, this length is $\xi_{\times} \sim\left(\sigma / \epsilon H_{p}\right)^{1 / 2} \sim \xi_{\times}\left(H_{p}\right)$. This question, as well as the appearance of $\xi_{\times}$itself, has not been addressed by previous models and experiments 24].

Within this region, there should be a crossover from the superrough interface with $\chi=1.25$ to a pinning regime. The roughness exponent on scales below the correlation length should then be determined solely by the local disorder configuration, i.e. be related to e.g. directed percolation depinning.

\subsection{Hydrodynamical description of Imbibition}

The model presented in Section 2 is purely diffusive and does not include any hydrodynamical modes. In principle, these could be incorporated, in a coarse-grained sense, along the lines of Refs. [33 and 48] by coupling the phase field to a velocity field described by the Navier-Stokes equations. Unfortunately, many problems need to be resolved before such an approach is taken, the main one consisting in establishing the role of hydrodynamics itself [24]. Contrary to other porous media like fractured rock or Hele-Shaw cell filled with glass beads, the paper matrix 
used in most imbibition experiments is not inert but often interacts strongly with the invading fluid through fiber swelling. Another complication arises from the transport of fluid through the paper. It is not at all obvious that an homogeneous pressure can be defined throughout the volume occupied by the fluid. In that sense, the model defined by Eq. (2) and Eq. (6) is the minimal model that includes a conservation law and, in conjunction with the appropriate set of boundary conditions, reproduces the experimental characteristic of imbibition.

Even though hydrodynamics is absent there is however of course a strong similarity between the model and the standard description of flow in porous media based on Darcy's Law for an incompressible fluid. In this description, the normal velocity of the interface is related to the gradient in the pressure field $P(\mathbf{x})$ by the permeability $\kappa$ as $v_{n}=-\kappa \partial_{n} P(x, y=h(x, t))$. The pressure is determined from Laplace's equation, together with $P(x, y=0)=P_{0}$, the atmospheric pressure, and a GibbsThomson boundary condition at the interface: $P(x, h)=$ $P_{0}-P_{c}(x, h(x, t))-\gamma \nabla^{2} h(x, t)$, which introduces a coarsedgrained surface tension [34] and capillary pressure $P_{c}$ arising from the microscopic menisci at the fluid-gas interface. Working to linear order in the small fluctuations of the interface, it is straightforward to find the pressure field, defined for $y \leq h(x, t)$,

$$
P(x, y)=P_{0}-\bar{P}_{c} \frac{y}{H}+\sum_{k} e^{i k x} \sinh (k y) P_{k},
$$

with the coefficient

$$
P_{k}=\frac{1}{\sinh (k H)}\left(\left(\gamma k^{2}-\frac{\bar{P}_{c}}{H}\right) h_{k}-P_{c}(k \neq 0, H)\right),
$$

where $\bar{P}_{c}$ represents the average capillary pressure and $P_{c}(k \neq 0, H)$ the fluctuations around it. It is then a simple matter to derive the interface equations, Eq. (13) and Eq. (14) in the appropriate limits. The derivation of the length scale $\xi_{\times}$may be transposed directly to the fluid imbibition case. This is essentially equivalent to the approach of Ref. [21] (see also [17]), although spontaneous imbibition requires a special treatment of the boundary conditions, absent in their work.

Our model is based on a constant mobility, which in the general case should be replaced by $\partial_{t} \phi=\nabla M(\mathbf{x}) \nabla \mu(\mathbf{x}, t)$. In spite of its simpleness, it is quite reasonable to ask whether the quenched randomness should not be included in the mobility, to model an effective quenched permeability. In most cases of forced fluid flow in bulk random media, this is where the non-uniformities are most relevant [4]. One point must however be emphasised. In any imbibition experiment designed for this purpose, the flow will never be large, and we believe that the random capillary forces will have the dominant influence. On the other hand, it has recently been shown that the presence of ink, or presumably of any other blocking material does make a quenched porosity relevant [31], a case which is not considered here.

It should also be pointed out that the field $\phi$ does not represent a real fluid density. In particular, any "air bub- bles" (i.e. connected regions of value $\phi=-1$ ) trapped behind the front will eventually dissolve. This is of course highly unrealistic for bulk porous media, but may be appropriate for thin porous media, where air can escape through surface pores.

\section{Conclusion}

In conclusion, a simple phase field model for the invasion of a liquid into a disordered system has been introduced. Liquid conservation is explicitly included. Some basic features observed in imbibition experiments are reproduced. Of course, it cannot account for many phenomena of paper wetting or invasion into porous media, which are briefly discussed in Section 1 and treated in more detail in the previous paper 24].

In numerical simulations, a superrough interface is found, with anomalous scaling due to a global roughness exponent $\chi \simeq 1.25>1$. A hardly discernible inherent anomaly of the structure factor may also be present. The extent of the spatial fluctuations of the interface are bounded by a length scale $\xi_{\times} \sim(\sigma H / \bar{\alpha})^{1 / 2}$, both in the freely rising and stationary imbibition setup. Interface fluctuations of a rising front are quasistationary, in the sense that at any time $t$ they are the same as in a stationary front kept at average height $H=H(t)$. The temporal fluctuations show multiscaling, which indicates motion by avalanches. The length and time scales where fluctuations saturate can be understood by simple dimensional considerations.

In relation to experiments, according to this analysis it is highly desirable to have fluids with small capillary pressure and high surface tension in order to obtain scaling over a large spatial regime. It would also seem appropriate to use organic liquids in experiments done with paper. These have minimal chemical interaction with the constituent fibers, and a simplified Washburn description of imbibition may be applicable. Another option is to use deionised water [31], again with the goal of reducing the fiber-liquid interaction. Above all, the main conclusion of the present work is that the macroscopic behaviour of the average interface position (i.e. $H(t)$ in a freely rising study, or $H(v)$ if the interface is stationary) is crucial to an understanding of the microscopic fluctuations of the interface, since it controls the range over which scaling can be observed.

\section{Acknowledgements}

We are grateful for discussions with and comments from R. Cuerno, R. Hilfer, V. Horváth, V. Kiiski, J. Krug, E. Seppälä and M. Smock. This work was supported by the Academy of Finland and the Research Corporation under grant No. CC4787 (KRE). S. M. acknowledges travel grant from the Magnus Ehrnrooth Foundation.

\section{Appendix}




\section{A Refining the Phase Field Model}

In this appendix, a generalised imbibition model is introduced. It rests on the following dynamical equation for the dimensionfull phase field $\tilde{\phi}(\mathbf{r}, t)$

$$
\frac{\partial \tilde{\phi}(\mathbf{r}, \tau)}{d \tau}-\tilde{G} \frac{\partial \tilde{\phi}(\mathbf{r}, t)}{\partial y}=\nabla M(\tilde{\phi}) \nabla \frac{\delta F}{\delta \tilde{\phi}}-\tilde{\epsilon}\left(\phi_{e}+\tilde{\phi}(\mathbf{r}, \tau)\right)
$$

with the free energy functional

$$
F=\frac{1}{2} \int d \mathbf{r}\left[r \tilde{\phi}^{2}+\frac{u}{2} \tilde{\phi}^{4}+\kappa(\nabla \tilde{\phi})^{2}-\tilde{\alpha} \tilde{\phi}\right]
$$

and $\phi_{e}=(r / u)^{1 / 2}$. For $\tilde{G}=0$ and $\tilde{\epsilon}=0$, as well as for constant mobility $M(\tilde{\phi})=M$, this reduces to the model described in the introduction. When $\epsilon \neq 0$, a non-conserving term is introduced in the equation of motion, which, in a first approximation describes evaporation of liquid (the $\tilde{\phi}=\phi_{e}$ phase), at a rate $2 \phi_{2} \epsilon$ and proportional to the total area covered by the fluid. The convective term is included to describe gravity. Although gravity can only be introduced properly through an hydrodynamical field 33,48 , it is shown below that this term reproduces the correct equation of motion for the average position of the imbibition front.

Assuming a constant mobility $M$, these equations can be put in a dimensionless form by defining

$$
\begin{array}{ll}
\mathbf{x}=\mathbf{r} / \zeta ; & \alpha=\left[\frac{u}{r^{3}}\right]^{1 / 2} \tilde{\alpha} ; \\
t=\left[\frac{M r^{2}}{\kappa}\right] \tau ; & G=\left[\frac{\kappa}{r^{3}}\right]^{1 / 2} \frac{\tilde{G}}{M} ; \\
\phi=\frac{\tilde{\phi}}{\phi_{e}} ; & \epsilon=\frac{\kappa}{M r^{2}} \tilde{\epsilon} .
\end{array}
$$

The ratio $\zeta=(\kappa / r)^{1 / 2}$ determines the width of the interfaces between different phases.

For now, let us concentrate on the case $G=\epsilon=0$. In one dimension, with $\bar{\alpha}$ being constant, the chemical potential obeys a Laplace equation in both wet and dry phases (actually there are minor corrections) with boundary conditions, $\mu(y=0)=0$ and $\mu(y=H)=\mu\left(y=L_{y}\right)=-\bar{\alpha}$, where $H$ is the position of the wet/dry interface and $L_{y}$ is the length of the paper. Thus

$$
\mu(y)= \begin{cases}-\bar{\alpha} y / H, & \text { if } y \leq H \\ -\bar{\alpha}, & \text { if } y>H\end{cases}
$$

which implies that $\phi$;

$$
\phi(y)= \begin{cases}\phi_{o}+\left(1-\phi_{o}\right) y / H, & \text { if } y \leq H \\ -1, & \text { if } y>H\end{cases}
$$

where $\phi_{0}>1$ is the solution of $-\phi+\phi^{3}=\bar{\alpha}$. In a first approximation, both $\phi$ and $\mu$ are linear functions of $y$ for $y \leq H$. The total amount of concentration is then

$$
\Phi_{t o t}(t)=\int_{0}^{L_{y}} \phi(y, t) d y=\frac{1}{2}\left(\phi_{o}+3\right) H(t)-L_{y},
$$

and the equation of motion for $\Phi_{t o t}$ is

$$
\frac{d \Phi_{t o t}}{d t}=\frac{\phi_{o}+3}{2} \frac{d H}{d t}=\int_{0}^{L_{y}} \frac{\partial^{2} \mu}{\partial y^{2}} d y
$$

or

$$
\frac{d H(t)}{d t}=\frac{2}{3+\phi_{0}}\left(\frac{\bar{\alpha}}{H(t)}\right)
$$

an equation similar to Washburn's result. Since $\phi_{0} \sim 1+$ $O(\bar{\alpha})$, this is actually Eq. (5) of Section 2. In presence of gravity and/or evaporation, the solution is more involved. As a first approximation, the difference between $\phi_{0}$ and unity is neglected and the Poisson equation for the chemical potential is considered,

$$
\frac{d^{2} \mu}{d y^{2}}-\epsilon=0
$$

for $y \leq H(t)$ and boundary conditions $\mu(y=0)=0$ and $\mu(y=H(t))=-\bar{\alpha}$. Again, $\mu(y>H(t))=-\bar{\alpha}$. The solution of Eq. (34) is

$$
\mu(y, H(t))=-\frac{\bar{\alpha} y}{H(t)}+\frac{1}{2} \epsilon y(y-H(t))
$$

Using the same procedure as above, the equation of motion of the interface is found to be

$$
\frac{d H(t)}{d t}=\frac{\bar{\alpha}}{2 H(t)}-G-\frac{1}{4} \epsilon H(t) .
$$

The "gravity" term acts exactly as in Washburn's equation in presence of gravity, and thus allows to identify $G$ as an effective gravity force acting on the interface. As far as we are aware, no detailed studies of fluid propagation in a thin porous medium with evaporation has been done.

Non-zero values of $G$ or $\epsilon$ will eventually stop the interface at an equilibrium height $H_{e q}=\bar{\alpha} / G$ if $G \gg \epsilon$ or $H_{e q}=(\alpha / \epsilon)^{1 / 2}$ if $\epsilon \gg G$. There is however a conceptual difference between pinning due to gravity or evaporation. In the former case, the chemical potential is a linear function of position, and pinning is determined by $\partial_{n} \mu\left(H_{e q}\right)=G$ while, in the latter case, the chemical potential is quadratic in $y$ and at pinning, $\partial_{n} \mu\left(H_{e q}\right)=0$. When both evaporation and pinning are present, the equilibrium height is determined by the zero of Eq. (36).

In terms of a dimensionfull interface height $\tilde{H}$, Eq. (36) becomes

$$
\frac{d \tilde{H}(\tau)}{d \tau}=\frac{1}{2} \frac{M \tilde{\alpha}}{\phi_{e}} \frac{1}{\tilde{H}(\tau)}-\tilde{G}-\frac{1}{4} \tilde{\epsilon} \tilde{H}(\tau)
$$

where $\tilde{\alpha}$ represents the average value of the disorder. The motion of the average interface in the pure Washburn case is thus determined by a combination of the mobility $M$ and the shift in the average chemical potential $\tilde{\alpha}$. The length scale $\xi_{\times} \sim(\tilde{\sigma} \tilde{H} / \tilde{\alpha})^{1 / 2}$ where now, $\sigma=$ $(2 \sqrt{2} / 3)\left(\kappa r \phi_{e}^{4}\right)^{1 / 2}$ is the dimensionfull surface tension. 


\section{B Projection to an Interface Equation}

To extract the interface equation Eq. (9) in the limit $G=0$ and $\epsilon=0$, the dynamical phase field equation must first be inverted with the use of the Green's function defined by Eq. (\$) and Eq. (10):

$$
\int d \mathbf{x}^{\prime} G\left(\mathbf{x} \mid \mathbf{x}^{\prime}\right) \frac{\partial \phi\left(\mathbf{x}^{\prime}, t\right)}{\partial t}=\mu(\mathbf{x}, t)
$$

It is then convenient to use a local coordinate system $(u, s)$ [50]. The 2-dimensional space is spanned by the the vector $\mathbf{x}(u, s)=\mathbf{X}(s)+u \hat{\mathbf{n}}(s)$, where $\mathbf{X}(s)$ is a point of the interface, $\hat{\mathbf{n}}$ is a unit vector normal to the interface and $s$ is the arc-length coordinate. In terms of the phase field, this corresponds to $\phi(u=0, s)=0$. The time derivative of the field then becomes $\partial \phi(u, s, t) / \partial t=V_{n}(s) \partial \phi / \partial u$ where $V_{n}(s)$ is the normal velocity of the interface at position $s$. If the interface (of thickness $\zeta=1$ in dimensionless units) is much smaller than the typical radii of curvature of the interface (the sharp interface limit), the Laplacian term of the chemical potential may be expanded such that

$$
\nabla^{2}=\frac{\partial^{2}}{\partial u^{2}}+\frac{\partial^{2}}{\partial s^{2}}+\mathcal{K}(s) \frac{\partial}{\partial u}
$$

where $\mathcal{K}(s)$ is the curvature of the interface. For $\alpha=0$, the one-dimensional kink solution is $\phi(u, s)=\phi_{0}(u)=$ $\tanh (u / \sqrt{2})$. The corrections to this form, represented by Eq. (30) are of order $\zeta \bar{\alpha} / H$. To first order thus, $\mu \sim$ $-\alpha(u, s)-\mathcal{K}(s) \partial \phi_{0}(u) / \partial u$. Still in the sharp interface limit $\zeta \mathcal{K} \ll 1$, the derivatives of the kink solutions have properties $\partial \phi_{0}(u) / \partial u \sim \Delta \phi \delta(u)$ and $\sigma=\int d u\left(\partial \phi_{0}(u) / \partial u\right)^{2}$ where $\Delta \phi \sim 2$ is the miscibility gap and $\sigma$ is the interface tension. Multiplying Eq. (38) by $\int d u\left(\partial \phi_{0}(u) / \partial u\right)$ then effectively project the phase field dynamics onto the interface $u=0$. A translation $u \rightarrow u+h(s, t)$ then yields

$$
\int d s^{\prime} G\left(s, h(s, t) \mid s^{\prime}, h\left(s^{\prime}, t\right)\right) V_{n}\left(s^{\prime}\right)=\eta(x, h(x, t))+\sigma \mathcal{K} .
$$

Equation (9) is then obtained by a further change of coordinate $s \rightarrow x$ and the relation $d s^{\prime} V_{n}\left(s^{\prime}\right)=d x^{\prime} \partial_{t} h\left(x^{\prime}, t\right)$.

\section{References}

1. For a recent overview see: J. Krug, Adv. Phys. 46, 139 (1997).

2. See e.g.: M. Kardar, Physics Reports 301, 85 (1998).

3. M.A. Rubio, Phys. Rev. Lett. 63, 1695 (1989).

4. V.K. Horváth, F. Family, and T. Vicsek, J. Phys. A 24, L25 (1991).

5. S. He, G.L.M.K.S. Kahanda, and P.-z. Wong, Phys. Rev. Lett. 69, 3731 (1992).

6. T. Delker, D.B. Pengra, and P.-z. Wong, Phys. Rev. Lett. 76, 2902 (1996).

7. A. Dougherty and N. Carle, Phys. Rev. E 58, 2889 (1998).

8. S.V. Buldyrev, A.-L. Barabási, F. Caserta, S. Havlin, H.E. Stanley and T. Vicsek, Phys. Rev. A 45 R8313 (1992).
9. A.-L. Barabási, S.V. Buldyrev, S. Havlin, G. Huber, H.E. Stanley, and T. Vicsek, in Surface Disordering: Growth, Roughening and Phase Transitions, R. Jullien, J. Kertész, P. Meakin and D.E. Wolf (Nova Science, Commack, 1992).

10. F. Family, K.C.B. Chan, J.G. Amar, in Surface Disordering : Growth, Roughening and Phase Transitions, R. Jullien, J. Kertész, P. Meakin and D.E. Wolf (Nova Science, Commack, 1992).

11. S.V. Buldyrev, A.-L. Barabási, S. Havlin, J. Kertész, H.E. Stanley, and H.S. Xenias, Physica 191 A, 220 (1992).

12. L.A.N. Amaral, A.-L. Barabási, S.V. Buldyrev, S. Havlin, and H.E. Stanley, Phys. Rev. Lett. 72, 641 (1994).

13. V.K. Horváth and H.E. Stanley, Phys. Rev. E 52, 5166 (1995).

14. P.B.S. Kumar and D. Jana, Physica A 224, 199 (1996).

15. T.H. Kwon, A.E. Hopkins, and S.E. O'Donnell, Phys. Rev. E 54, 685 (1996).

16. T. Nagamine and S. Miyazima, Fractals 4, 293 (1996).

17. O. Zik, E. Moses, Z. Olami, and I. Webman, Europhys. Lett. 38, 509 (1997).

18. It should be noted that the scaling regime of Ref. [8] is confined to a length scale $l<1 \mathrm{~cm}$, i.e. a length scale of the order of the mean fiber length itself. Ref. [12] addresses this problem indirectly, but the "universality" of the scaling on such short length scales remains questionable.

19. J.P. Bouchaud and A. Georges, Phys. Rep. 195, 127 (1990).

20. A.E. Scheidegger, The Physics of Flow through Porous Media, MacMillan Co, New-York (1957).

21. V. Ganasan and H. Brenner, Phys. Rev. Lett. 81, 578 (1998).

22. E.W. Washburn, Phys. Rev. 17, 273 (1921).

23. See e.g. T. Gillespie, J. Coll. Science 13, 32 (1958), and ibid 14, 123 (1959); or E. Sissa, J. Colloid Interface Sci. 121, 22 (1988) and I. Pézron et al., J. Colloid Interface Sci. 173, 319 (1995) and refs. therein for more recent work.

24. M. Dubé, M. Rost and M. Alava, Submitted to Eur. J. Phys. (November 1999).

25. M. Dubé, M. Rost, K.R. Elder, M. Alava, S. Majaniemi, and T. Ala-Nissila, Phys. Rev. Lett. 83, 1628 (1999).

26. N. Provatas, M. Alava, and T. Ala-Nissila, Phys. Rev. E 54, 36 (1996).

27. J.W. Cahn and J.E. Hilliard, J. Chem. Phys. 28, 258 (1958).

28. B.I. Halperin and P.C. Hohenberg, Rev. Mod. Phys. 49, 435 (1977).

29. A.J. Bray, Adv. in Phys. 43, 357 (1994).

30. J.S. Langer, in Solid Far From Equilibrium, Ed. C. Godrèche, (Cambridge U.P., 1992).

31. O. Zik, T. Kustanovich, E. Moses, and Z. Olami, Phys. Rev. E 58, 689 (1998).

32. J.S. Langer and L.A. Turski, Acta Metall. 25, 1113 (1977).

33. K. Kawasaki and T. Ohta, Prog. Theo. Phys. 68, 129 (1982).

34. J. Krug and P. Meakin, Phys. Rev. Lett. 66, 703 (1991).

35. G.S. Bales and A. Zangwill, Phys. Rev. B 415500 (1990); W.K. Burton, N. Cabrera, and F.C. Frank, Phil. Trans. R. Soc. (London) A 243, 299 (1951).

36. S. Mukherji and S.M. Bhattacharjee, Phys. Rev. Lett. 79, 2502 (1997).

37. W.W. Mullins and R.F. Sekerka, J. Appl. Phys. 35, 444 (1964). 
38. H. Leschhorn, T. Nattermann, S. Stepanow, and L.-H. a) Tang, Ann. Physik 6, 1 (1997). (1993).

39. J.M. López, M.A. Rodríguez, and R. Cuerno, Phys. Rev. E 56, 3993 (1997).

40. H. Leschhorn and L.-H. Tang, Phys. Rev. Lett. 70, 2973 (1993).

41. J. Krug, Phys. Rev. Lett. 72, 2907 (1994).

42. H. Leschhorn and L.H. Tang, Phys. Rev. E 49, 1238 (1994).

43. K. Sneppen, Phys. Rev. Lett. 69, 3539 (1992).

44. Z. Olami, I. Procaccia and R. Zeitak, Phys. Rev. E 49, 1232 (1994).

45. H. Leschhorn, Physica A 195, 324 (1993).

46. S. Roux and A. Hansen, J. Phys. I France 4, 515 (1994).

47. M. Jost and K.D. Usadel, Phys. Rev. B 54, 9314 (1996).

48. D. Jasnow and J. Viñals, Phys. of Fluids 7, 747 (1996).

49. A. Koponen, D. Kandhai, E. Hellén, M. Alava, A. Hoekstra, M. Kataja, K. Niskanen, P. Sloot, and J. Timonen, Phys. Rev. Lett. 80, 716 (1998).

50. W. van Saarloos, Phys. Rep. 301, 9 (1998).

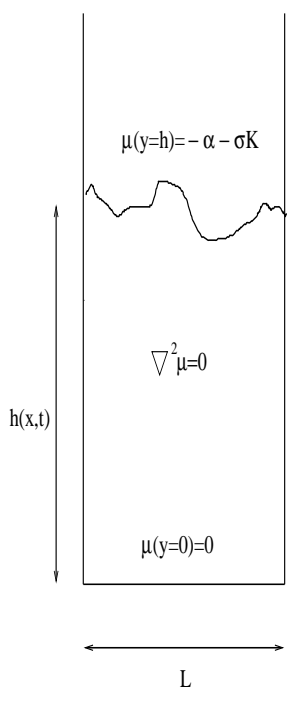

b)
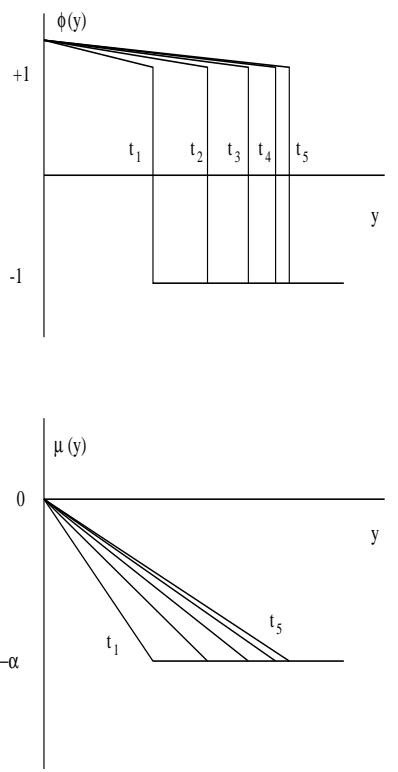

Fig. 1. Setup of the imbibition model. The system is defined on the plane $y>0$, with lateral extent $L$. The average position of the interface is represented by $H(t)$. The chemical potential obeys Laplace's Equation in the bulk $\nabla^{2} \mu=0$ with GibbsThomson boundary condition $\mu=-\alpha(\mathbf{x})-\sigma \partial_{x}^{2} h(x, t)$ at the interface and the imposed value $\mu(y=0)=\alpha_{0}=0$ at the bottom.

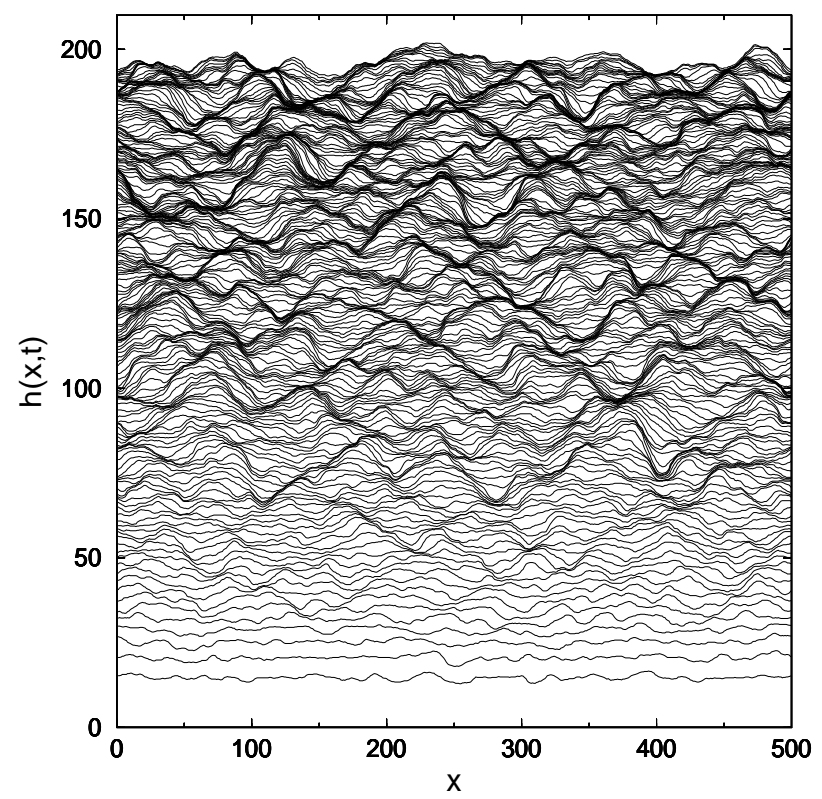

Fig. 2. Front configurations of a rising interface at equal time intervals $\Delta t=10^{3}$. Their average separation becomes smaller as the front slows down due to the conservation law. 


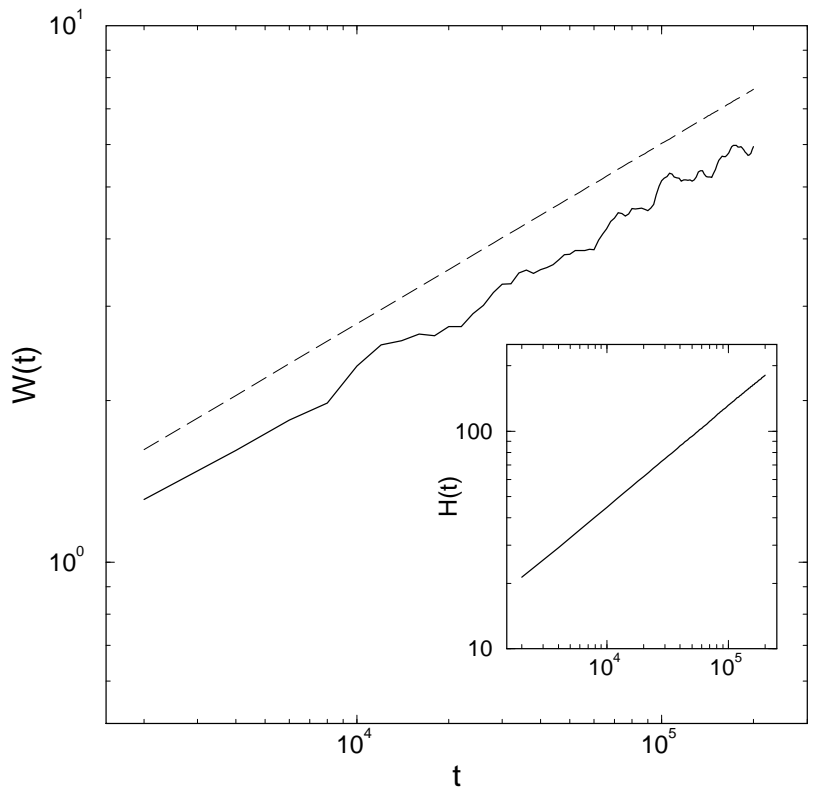

Fig. 3. Increase of the width of the interface as a function of time. The data are for a system of lateral extent $L=256$, with $\bar{\alpha}=0.2$ and $\Delta \alpha=0.2$. The straight dashed line has slope $\beta=0.32$. The inset shows the average position of the interface $H^{2}(t)=\bar{\alpha} t$, in agreement with Eq. (9).

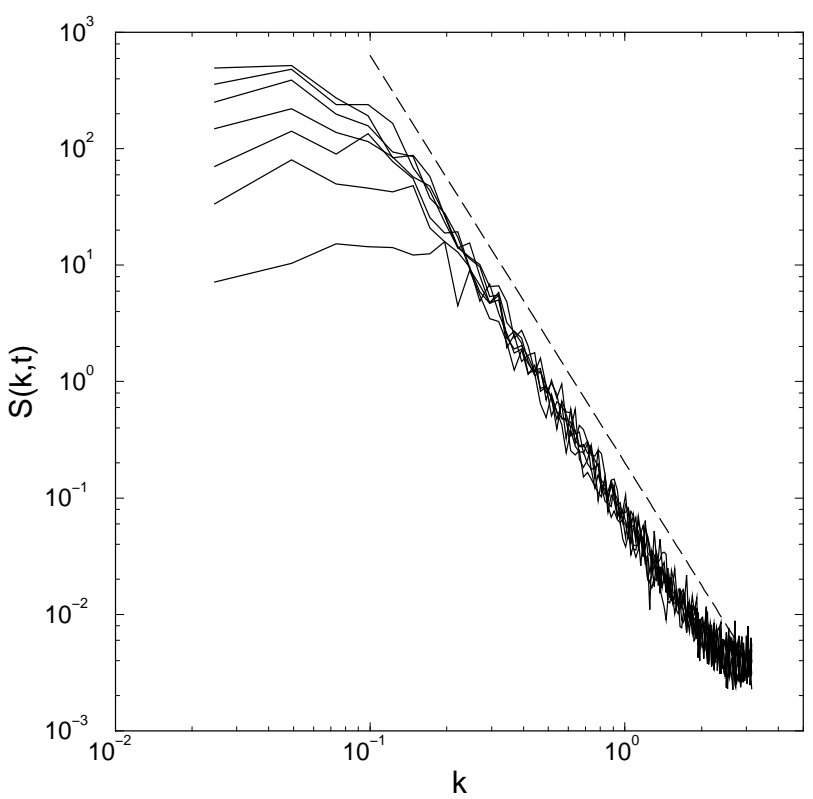

Fig. 4. Structure factor for a system with $L=256, \bar{\alpha}=$ $\Delta \alpha=0.2$ at different times. The data are for times $t=5 \times 10^{3}$ (lowest curve) to $t=5 \times 10^{4}$ (upper curve) at intervals of $10^{4}$. The dashed line has a slope -3.5 indicating a global roughness exponent $\chi \sim 1.25$. A weak intrinsic anomalous scaling, in the sense of Ref. [39] may be present, but cannot be clearly identified.

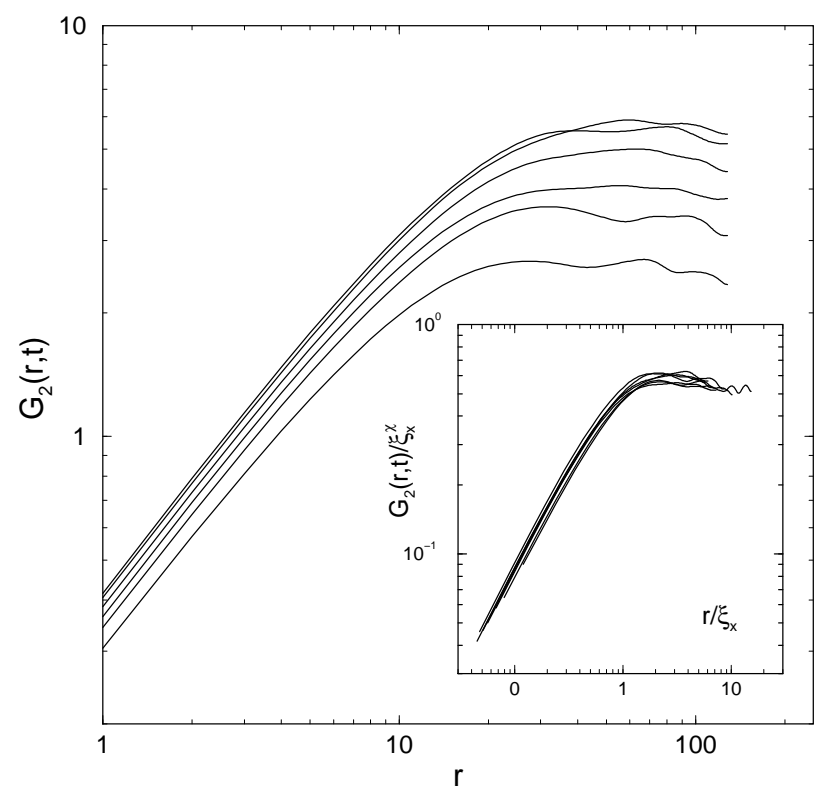

Fig. 5. Spatial correlation functions $G_{2}(r, t)$ for parameters identical to those of Fig. 1 . The anomalous scaling is indicated by the increase of the average mean step height $G_{2}(r=1, t)$ and the local exponent $\chi_{l 0 c} \sim 0.9$. In the inset, the data are rescaled according to Eq. (19). The scaling shows the existence of a lateral length scale $\xi \sim t^{1 / 4}$.

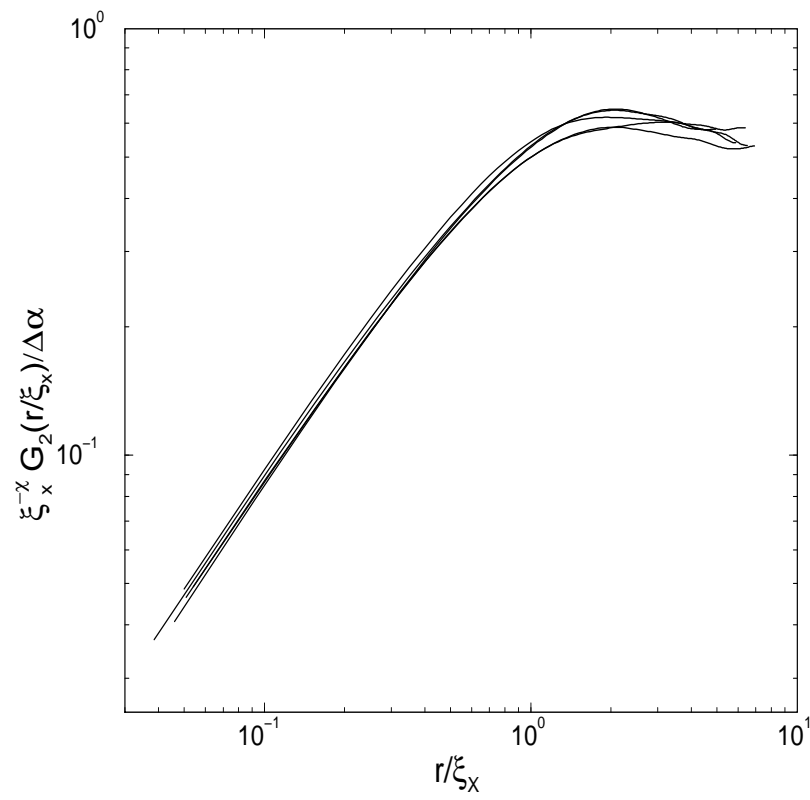

Fig. 6. Plot of the correlation functions $G_{2}(r, t)$ according to the scaling form Eq. (19). The data are taken from systems with lateral size $L=256$ and gaussian distribution, with parameters (i) $\bar{\alpha}=0.05, \Delta \alpha=0.2$, at height $H=20$, (ii) $\bar{\alpha}=0.1, \Delta \alpha=0.2$, at height $H=67$, (iii) $\bar{\alpha}=0.2, \Delta \alpha=0.2$, at height $H=77$, (iv) $\bar{\alpha}=0.3, \Delta \alpha=0.2$, at height $H=102$, (v) $\bar{\alpha}=0.2, \Delta \alpha=0.1$, at height $H=95$. 


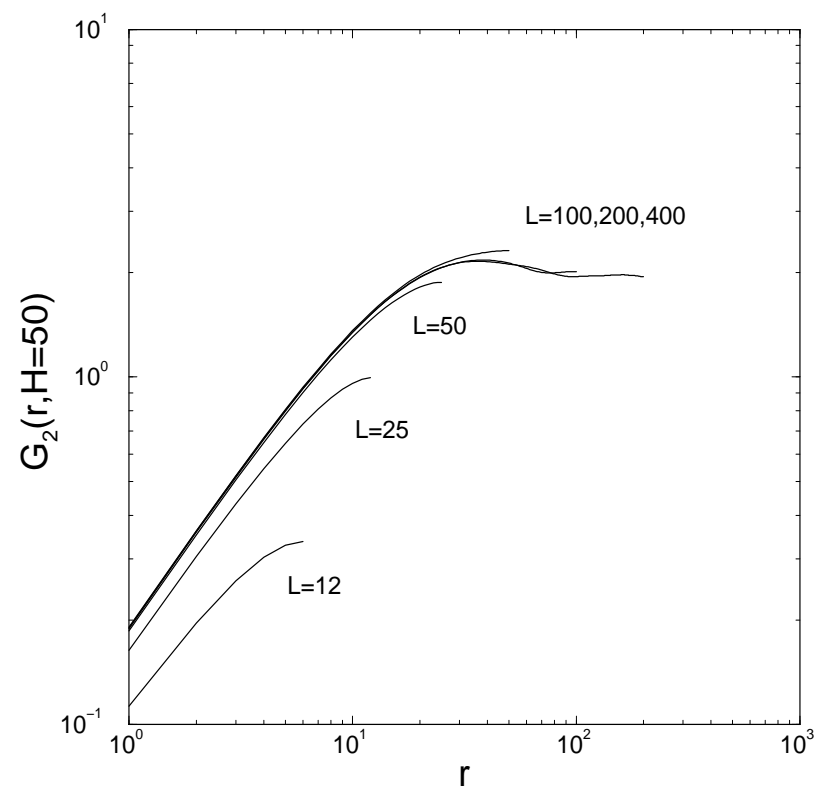

Fig. 7. Spatial height difference correlation functions for a setup with fixed average height $H=50$, and $\alpha(\mathbf{x})$ uniformly distributed on the range $[0,0.4)$. The data for $L=100,200$ and 400 fall together (top curves), while small systems show $L$ dependence ( $L=50,25$ and 12 , middle to bottom).

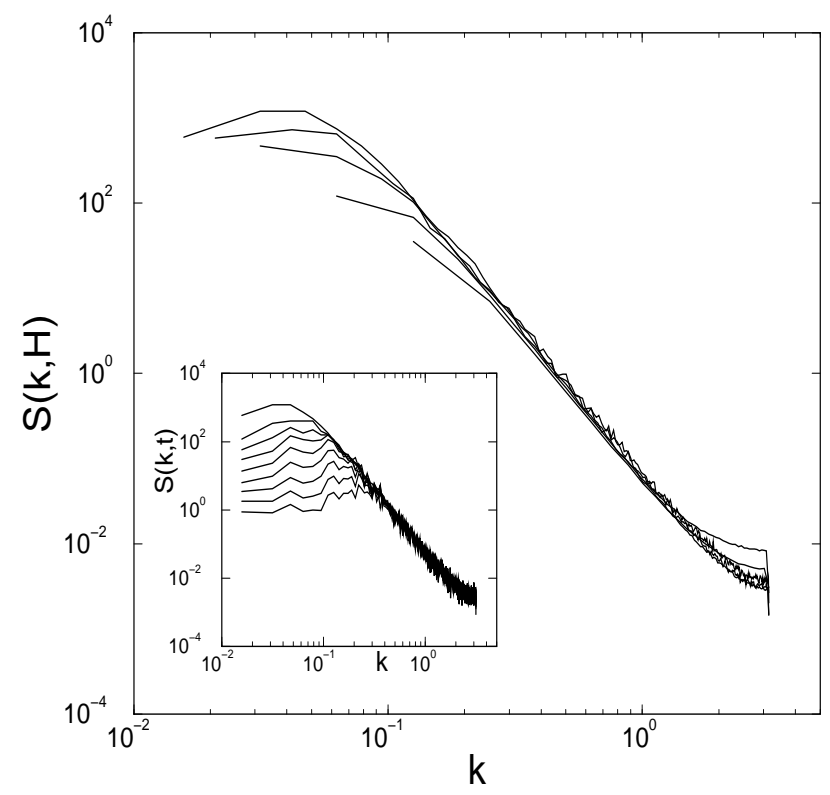

Fig. 8. Structure factors $S(k)$ for $H=25,50,100,150,200$ (bottom to top) and $L=2 H$, disorder chosen from an exponential distribution with $\bar{\alpha}=0.2$. The power law decay is proportional to $k^{-3.5}$, indicating a global roughness exponent $\chi=1.25$. The large scale cutoff $k^{*} \sim 1 / \xi_{\times}$decreases with $H$. The inset shows the approach of $S(k, t)$ to the saturated $S(k)$ in the system of size $L=2 H=400$ and for times $t=2^{n} \times 10^{2}$ with $n=0,1, \ldots 7$.

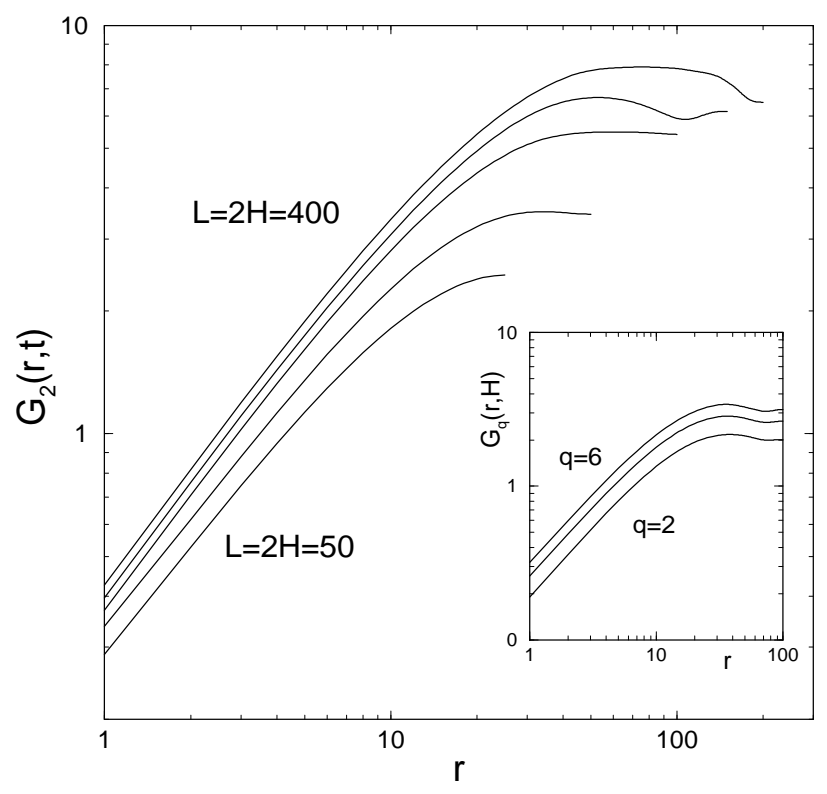

Fig. 9. Correlation function $G_{2}(r)$ for the same data as in Fig. 8. The curves saturate at a length $\xi_{\times}$which increases with $H$, together with the "step height" $G_{2}(r=1)$. The local scaling exponent $\chi_{\text {loc }} \approx 0.95$, close to the expected value $\chi_{\text {loc }}=1$. In inset, the higher moments of the correlation function $G_{q}(r, H)$ are shown for a system with $L=200$ and $H=50$. All moments have the same local exponent $\chi_{l o c, q} \sim 1$.

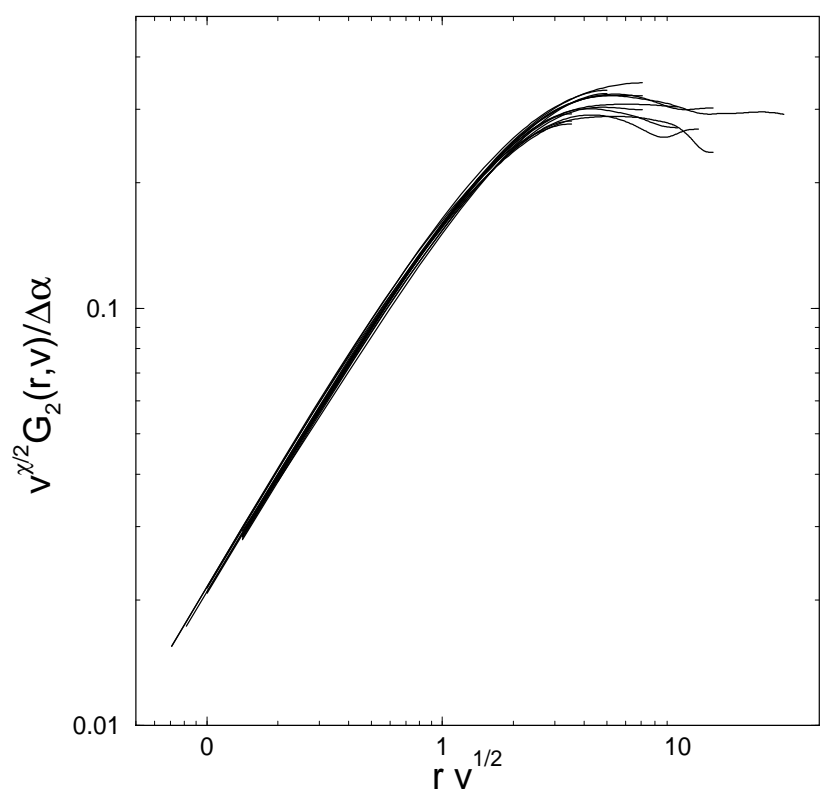

Fig. 10. Scaling plot of $G_{2}(r, v) /\left(\Delta \alpha \xi_{\times}^{\chi}\right)$ vs. $r / \xi_{\times}$with global roughness exponent $\chi=1.25$ and $\xi_{\times} \sim v^{-1 / 2} \sim H^{1 / 2}$. A wide range of $H$, from 25 to 400 , and all three forms of disorder are used. The data compare well to the scaling relations derived in Eq. (21). 


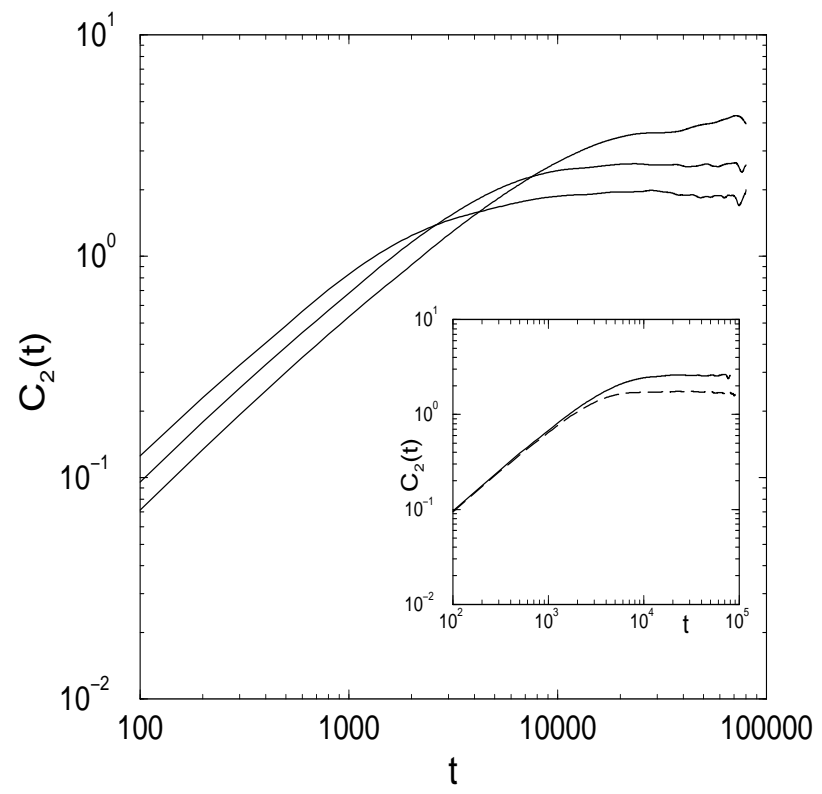

Fig. 11. Correlation functions $C_{2}(t)$ for $L=400$ and $H=$ $50,100,200$ (solid curves). The crossover time $t_{s}$ and the level of saturation increase with $H$. At short times $C_{2}(t)$ decreases with $H$. In inset, $C_{2}(t)$ for $L=50$ and $H=100$ (dashed line) is compared to $C_{2}(t)$ for $L=400$ and $H=100$ (solid curve). The transition to the saturated regime is sharper for smaller $L$.

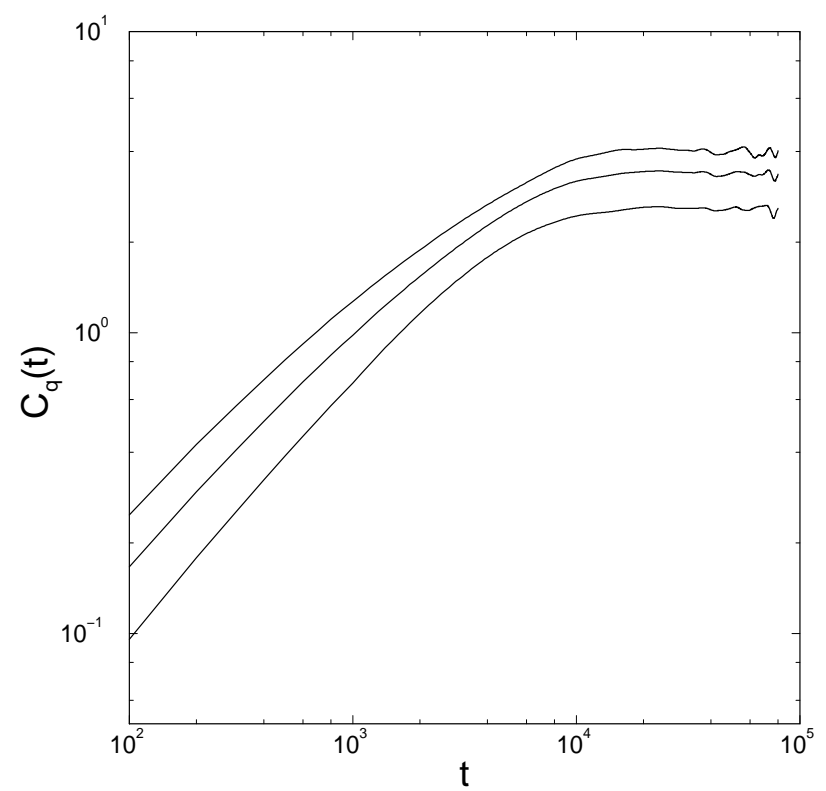

Fig. 12. Correlation functions $C_{q}(t)$ for $q=2,4$ and 6 for systems of size $L=200$ and $H=50$, such that $L>\xi_{\times}$. Each moment increases with a different exponent $\beta_{q}$.

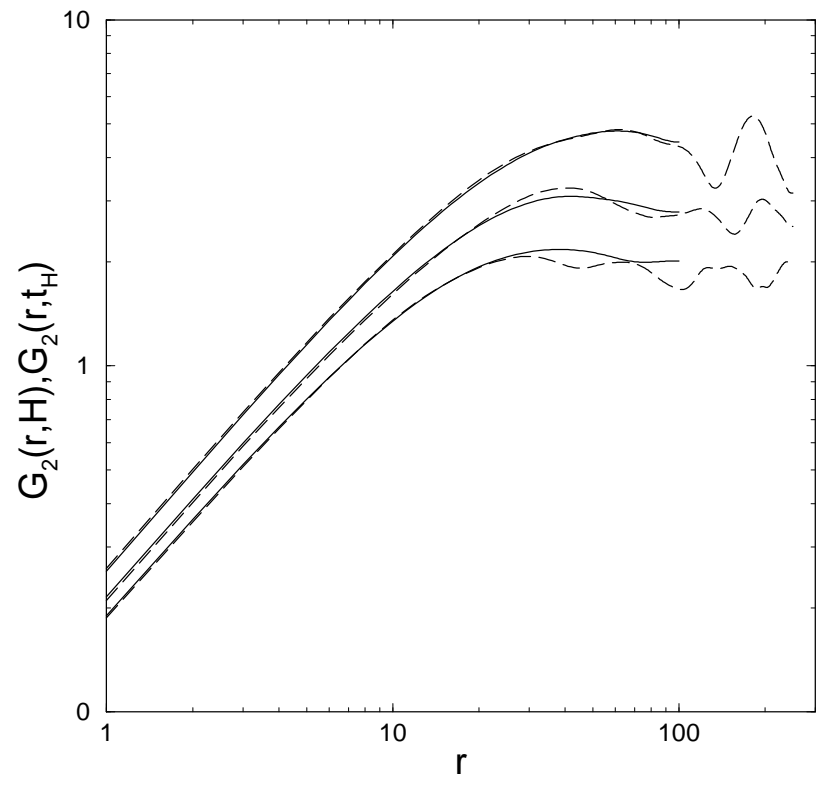

Fig. 13. Comparison of the correlation function $G_{2}(r, H)$ in the stationary setup (solid lines) at heights $H=25,50$ and 100 to $G_{2}\left(r, t_{H}\right)$ in the freely rising case (dashed lines) at corresponding times $t_{H}=H^{2} / \bar{\alpha}$. There is a complete equivalence between both situations. 